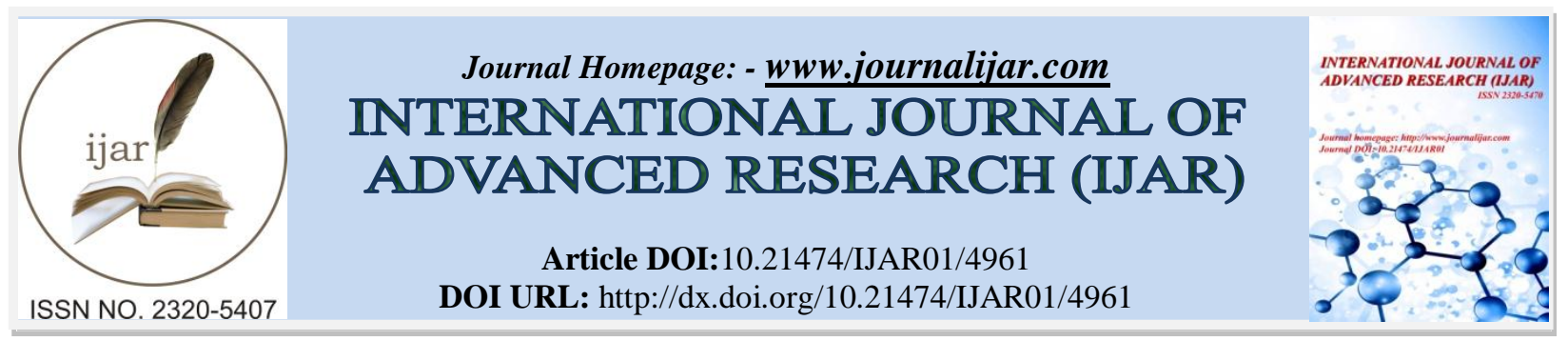

RESEARCH ARTICLE

\title{
OXIDATIVE STRESS, ANTIOXIDANT SCAVENGING SYSTEMS AND DIABETES MELLITUS: A CONCISE REVIEW.
}

Farah Jabeen, Farha Aziz and Husan Afroz Rizvi.

Department of Biochemistry, Jinnah University for Women, Karachi - Pakistan.

\section{Manuscript Info}

\section{Manuscript History}

Received: 26 May 2017

Final Accepted: 28 June 2017

Published: July 2017

Key words:-

Oxidative stress; ROS, antioxidants, lipid peroxides, free-radical, diabetes mellitus

\section{Abstract}

The enormous majority of complex life on earth requires oxygen for its survival. Oxygen is an extremely reactive molecule that damages living organisms by producing reactive oxygen species leading to oxidative stress. Oxidative stress arrived from increasing amount of ROS or as a consequence of increased levels of lipid peroxides and free-radical intermediates, as well as the reduced total antioxidant capacity that may cause the reduction of molecular oxygen or oxidation of water to leads to the formation of free radicals that could damage cellular lipids, membranes, proteins and DNA (Rains et al, 2011). Living organisms have a complex network of antioxidant metabolites and enzymes that work mutually to prevent oxidative damage to cellular components Ido et al, 1997). Advanced oxidative stress and alterations in antioxidant potential, observed in both clinical and investigational diabetes mellitus. Alteration in oxidative stress biomarkers, including Catalase, superoxide dismutase, glutathione, glutathione reductase, glutathione peroxidase, antioxidant vitamins, lipid peroxidation, non enzymatic glycosylated proteins, are helpful in identifying the risk of developing vascular complications in diabetics. For the period of the preceding few years, more attention has been gained on the involvement of oxidative stress in diabetes and it has been established that oxidative stress in association with nonenzymatic glycosylation of protein and glucose auto-oxidation might contribute in the pathogenesis of secondary complications of diabetes (Mullarkey et al, 1990; Ceriello et al, 2000). In this review article, we summarize the effect of oxidative stress in the development and pathogenesis of diabetes and its obstacle and the role of antioxidants in reducing oxidative stress and lessening of diabetic complications.

Copy Right, IJAR, 2017,. All rights reserved.

\section{Introduction:-}

The oxygen which is utilized during normal cellular metabolism for generating energy produces ROS. In physiological state, the superoxide anion is formed in various steps of the electron transport chain as a by-product, through the course of normal oxidative phosphorylation, which is essential for producing ATP. Moreover Peroxide is formed from the oxidation of reduced flavoproteins (Lenaz et al, 2001). These free radicals are counteracted by the body's self defense system. The ROS formed inside the cells are hydrogen peroxide $\left(\mathrm{H}_{2} \mathrm{O}_{2}\right)$, hypochlorous acid $(\mathrm{HOCl})$, and free radicals for example the hydroxyl radical $(\cdot \mathrm{OH})$ and the superoxide anion $(\mathrm{O} 2-)$. These oxidants 
can spoil the cells by initiating chemical chain reactions through lipid peroxidation, or by oxidizing DNA or proteins (Sakurai et al, 1988).

\section{Hyperglycemic induced Oxidative Stress in Diabetes:-}

Homeostasis of Glucose is retained by the well synchronized communication of three physiologic routes: insulin secretion, up taking of glucose by tissue and hepatic glucose production. The body strives to maintain a continuous delivery of glucose for providing energy to cells by keeping constant glucose concentration in blood. Usually glucose homeostasis corresponds to the equilibrium between intake (glucose assimilation from gut), tissue consumption (Glycolytic pathway, HMP shunt, TCA cycle and glycogen production) and endogenous production (gluconeogenesis and glycogenolysis) (Meyer et al, 2002). Failure to maintain this homeostasis results various disturbances in carbohydrate, protein and fat metabolism. Inadequate or inactive release of insulin or less utilization of glucose by cell leads to hyperglycemia, which if not treated, produce serious long term micro and macro vascular complications. Uncontrolled blood glucose level over a longer period results severe micro-vascular impediments including nephropathy, neuropathy and retinopathy (Yamagishi et al, 2005; Van Dam et al, 2002) as well as macrovascular complications such as cerebrovascular disease, cardiovascular and peripheral vascular disease (Grobbee et al, 2003; Thompson et al, 2008). It is reported that micro-vascular complications start developing at least 7 years prior to the confirmed diagnosis of type-2 diabetes (Harris et al, 1995). As no early symptoms of diabetes appear, most of the people remain unaware of the disease for many years. People usually consult physician when symptoms of hyperglycemia like polyurea, polydipsia, polyphagia, fatigue, blurred vision, unexplained weight loss, slow healing of wound, deadness (numbness) or itchy (tingling) in hands or feet and cardiovascular disease appear. The vascular complications are believed to be the major cause of morbidity and mortality in diabetic patients (Klein et al, 1995; Haffner et al, 1998; Patel et al, 2008 ) and hyperglycemia is supposed to be a major factor responsible for these complications. The mechanism, how these vascular diseases are produced, is still not clear. However, the evidences suggested, that hyperglycemia produce major disturbances in number of metabolic pathways leading to damages in vascular tissues. The most prominent pathways which indicate, that hyperglycemia promotes cellular dysfunctions include: the aldose reductase or Polyol pathway (Setter et al, 2003).AGE pathway (Brownlee et al 1998; Gillery et al, 2001), activation of protein kinase C (Xia et al, 1994; Inoguchi et al, 2003) and oxidative stress produced by enhanced production of ROS and defective defense system (Baynes et al, 1999; Vincent et al, 2004). The studies conducted by Diabetic Control and Complication Trial (DCCT) and the United kingdom prospective diabetes study (UKPDS) have undoubtedly demonstrated that improve glycemic control reduces the risk of development and progression of several micro and macro-vascular complications in diabetes (DCCT Research Group; 1993).

Hyperglycemia is unusually high blood glucose levels that take place when the body does not make adequate insulin or whenever the body can't utilize the produced insulin appropriately. It induces oxidative stress in diabetes (both in type 1 and type 2), either by the direct production of ROS or by changing the redox equilibrium in the cells. The redox balance can be altered in several ways such as, by raising the Polyol pathway flux, increasing the intracellular production of AGEs, activation of PKC or by the over-making of superoxide by mitochondrial electron transport chain (Rains et al, 2011).

In polyol pathway, reductions of glucose to sorbitol take place by means of aldose reductase with the help of NADPH. Resultant Sorbitol is oxidized to fructose and NADH is oxidized to NAD+ by sorbitol dehydrogenase. In the reaction, the aldose reductase reduces the toxic aldehydes produced by ROS or other substrates to inactivate alcohols. In hyperglycemia there is an improved production of sorbitol and an increased activity of aldose reductase, which decreased NADPH, an important cofactor in the production of GSH. GSH is an intracellular antioxidant that prevents the damages of cellular components by ROS. Hyperglycemia does not generate ROS directly, but promotes the redox inequity in the cell, leading to oxidative stress ${ }^{24}$. Hyperglycemia increases the synthesis of AGEs (advanced glycation end products); a variety of protein adducts whose accumulation has been concerned with tissue damage (Suzuki et al, 1999). The AGEs are created through covalent attachment of aldehyde or ketone moiety of reducing sugars to free amino groups of proteins, form a Schiff's base. The base will reorganize into an Amadori product, a very established ketoamine, which can directly be changed into AGEs. The AGEs are supposed to be concerned in the genesis of the irreparable complications of diabetes (Maritim et al, 2002).

Hyperglycemia contributes to the direct and indirect formation of ROS by stimulating DAG-PKC pathway. Protein kinase C (PKC) consists of isoforms of the protein and is activated by DAG, a lipid courier. PKC can be activated through attachment of AGE receptors, which in turn causes fluctuations in cell signaling. The over production of 
superoxide by mitochondrial ETC is a major factor in the production of ROS. Normally the glucose oxidation initiate in the cytoplasm, where glucose undergoes glycolytic pathway. During glycolysis NADH and pyruvate are formed, both of them provide electrons to the ETC for ATP production through chemical reactions. The electrons are then provided to molecular oxygen, which consecutively amplifies the mitochondrial superoxide production (Rains et al, 2011). Oxidative stress can stimulate the multiple genes expression including signaling molecules for example protein kinase $\mathrm{C}$, nuclear factor-B, and extracellular signal-regulating kinase in vascular cells, which may boost up atherogenesis (Mironova et al, 2000).

\section{Sources of Oxidative Stress in Diabetes:-}

There are several sources of oxidative stress in diabetes including non-enzymatic and enzymatic ways.

\section{Non-Enzymatic Sources:-}

Non-enzymatic basis of oxidative stress initiate from the oxidative biochemistry of glucose. In addition to the routine mitochondrial source other metabolic pathways also contributing towards the generation of ROS. The following are the major processes where excess glucose might be shunted into when it accumulates beyond the capacity of glycolytic enzymes, ultimately producing ROS (Sies et al, 1993).

\section{Glucose Autoxidation:-}

Hyperglycemia could directly caused amplification of ROS creation. Glucose can go through autoxidation and produce $\cdot \mathrm{OH}$ radicals through metal catalyzed redox reaction of hydrogen peroxide. Auto-oxidation of glucose is well-recognized to make oxygen-cored free radicals (Niedowicz et al, 2005).

\section{Protein Glycation:-}

Glucose reacts non-enzymatically with proteins causing the Amadori products development following the formation of heterogeneous class of compounds, together called as advanced glycation end products (AGEs). ROS is produced at various steps throughout this process. Excess glycemia accelerates this process of making (AGEs) in diabetes (Wolff et al, 1991).

\section{Sorbitol Pathway:-}

In hyperglycemia the glucose metabolism via polyol (sorbitol) pathway is greatly augmented, which in turn results in greater $\bullet \mathrm{O} 2$ formation (Johansen et al, 2005).

\section{Mitochondrial Source:-}

An additional source of non-enzymatic generation of reactive species is the mitochondrial respiratory chain. Oxidative phosphorylation during aerobic glycolysis produces ROS, a normal process that might become extreme in hyperglycemic states. It has been established that hyperglycemia-provoked production of $\bullet \mathrm{O} 2$ - at the mitochondrial stage, is the prime event in eliciting a violent cycle of oxidative stress in diabetes (Nishikawa et al, 2000). The major function of mitochondria is to supply energy for almost all cellular processes. Another important function of mitochondria is the regulation of insulin linked to the glucose level (Rains et al, 2011). ROS may damage some mitochondrial components including lipids, proteins and mitochondrial DNA which disturb mitochondrial normal functions and ultimately lead to insulin resistance. There are two possible ways of oxidative stress that gives insulin resistance. One of them is the increase of uncoupling protein 2 (UCP2), which is a protein on the internal membrane of mitochondria. When it is activated it causes protons to escape across the membrane and instead of the ATP production, heat is produced. This is a mitochondrial dysfunction which is going to reduce mitochondrial fatty acid oxidation, resulting increased fatty acyl-CoA and DAG. Serine- and threonine kinase are activated and hold back the glucose transport. The other way is, when oxidative stress directly reduces ATP that will decrease glucose transport and consequently cause insulin resistance (Rains et al, 2011).

\section{Enzymatic Sources:-}

Enzymatic sources of amplified generation of reactive species in diabetes comprise of Nitric oxide synthase (NOS), NADPH oxidase and Xanthine oxidase.

\section{Uncoupled Nitric Oxide Synthase (NOS):-}

Endothelial nitric oxide synthase (eNOS) is the enzyme which normally synthesizes $\bullet$ NO from L-arginine, that serve as substrate for the enzyme. There are five cofactors/prosthetic groups mandatory for all isoforms of NOS. If NOS 
be deficient in its substrate or one of its cofactors, it possibly will produce $\bullet \mathrm{O} 2$ - as a substitute of $\bullet \mathrm{NO}$. This is termed as the uncoupled condition of NOS (Guzik et al, 2002).

\section{NADPH Oxidase (Nox):-}

NADPH Oxidase has a committed role of producing ROS. Several evidences suggest that in cellular stress responses Nox has an imperative role in signal transduction. The system of Nox can be triggered by a collection of chemical, physical, and biological cellular stresses. In various condition, activation of Nox happen in response to the cellular stress paradigm, in the sense that the response can initiate by verity of cellular stresses, stimulation of mitogenactivated protein kinases by Nox-derived ROS and involvement of Nox in stress cross-tolerance development. Nox may also convey signals in the direction of apoptosis in irreversibly injured cells. Following injury at later phase, Nox is concerned with tissue repair by transforming cell proliferation and fibrosis and angiogenesis. It has been idealized that Nox might have a fundamental role in cell stress responses and the successive tissue repair (Jiang et al, 2011).

Xanthine Oxidase (XOD):-

Xanthine oxidase is an oxidoreductase enzyme that creates ROS. It catalyzes hypoxanthine to xanthine oxidation and can additionally catalyze the xanthine to uric acid oxidation. XOD enzyme actively participates in the catabolism of purines in some species, together with humans (Hille et al, 2005; Harrison et al, 2002). Xanthine oxidase inhibition has been projected as a mechanism for improving cardiovascular health state (Dawson et al, 2006).

\section{Cyclooxygenase (COX):-}

Cyclooxygenase also known as prostaglandin-endoperoxide synthase (PTGS), is responsible for synthesis of important biological mediators collectively known as prostanoids (prostaglandins, prostacyclin and thromboxane). The formation of prostaglandins, levuloglandins and thromboxane, is catalyzed by the cyclooxygenase isoenzymes COX-1 and COX-2. The prostaglandins are important mediators that affect almost all known physiological and pathological processes by means of their reversible interaction with G-protein coupled membrane receptors. The pyretic, inflammatory, thrombotic, oncological and neuro-degenerative diseases can be relieved by inhibition of COX (Fitzpatrick et al, 2004).

\section{Metabolic Alterations Caused by Oxidative Stress and their Deleterious Effects:-}

Metabolic derangements are related with diabetes mellitus including hyperglycemia, AGEs, amplified intensities of FFAs, and lipoprotein aberrations, such variation have been found in people with type 1 and type-2 diabetes (Bierhaus et al, 1998). Metabolic alterations caused by oxidative stress and their harmful effects are as follows:

\section{Lipid Peroxidation:-}

In oxidative stress, ROS may enhance the oxidation of LDL. The oxidized LDL thus produced, is not recognized by LDL receptor and can be use by scavenger receptors in macrophages directs to foam cell formation and atherosclerotic plaques (Boullier et al, 2001). ROS-provoke membrane peroxidation of lipids transforms the biological membranes structure and the fluidity, which ultimately affects its roles. All these modifications involve in the pathogenesis of vascular dysfunction (Davi et al, 2005).

\section{Advanced Glycation End Products (AGEs):-}

Reactive glycation precursors, both endogenous or exogenous, assault proteins and lipids to form multifaceted and irreversible substances, which are extremely harmful to the vessel wall integrity and function (Baynes et al, 1991).

This can happen in a number of ways:-

$>$ AGE cross-bridges (formed between the macromolecules) mediated Mechanical dysfunction

$>$ The enhance accumulation of AGE into the vessel wall by means of catching blood components, e.g., lipoproteins, immunoglobulins and cells (including platelets and nitrous oxide derivatives).

$>$ Wide range of modification in cell function, that appears to engage in receptor and non-receptor pathways (Bierhaus et al, 1998).

\section{Oxidation in DNA:-}

Like oxidation of proteins and lipids, DNA can also be oxidized. If there is an elevated rate of ROS, there is a possibility of abnormally high DNA oxidation, which is exhibited in many diabetic patients. It is essential to 
examine the DNA oxidation because of its impact on mitotic tissue. The increased DNA oxidation can trigger DNA mutation which may develop into cancer (Pazdro et al, 2010).

\section{Natural Defense System against Oxidative Stress:-}

Oxidation reactions can generate free radicals that possibly will initiate chain reactions that cause damaging of cells. In general, antioxidant systems either avert these reactive species from being produced, or eliminate them prior to their damaging impacts on cell (Sies et al, 1997).As reactive oxygen species do have valuable roles in cells, for instance redox signaling, the antioxidant systems do not eliminate oxidants totally, but maintain them at an optimum level (Rhee et al, 2006). A diversity of antioxidants (endogenous and exogenous), work together inter relatively and synergistically to counteract free radicals. Some of the prominent antioxidants are described as under:

- Nutrient-derived Antioxidants such as ascorbic acid (vitamin C), tocopherols (vitamin E), carotenoids, and additionally low molecular mass compounds such as glutathione and lipoic acid.

- Antioxidant Enzymes catalyzing free radical turn off reaction e.g., catalase, superoxide dismutase, glutathione reductase and glutathione peroxidase.

- Metal-binding Proteins including albumin, ferritin, lactoferrin, and ceruloplasmin that eradicate free iron and copper ions which catalyze oxidative reactions. These are preventing antioxidants that hold back the formation of free radicals (Jacobet al, 1995).

\section{Antioxidant Metabolites:-}

Antioxidants are classified into two wide categories, hydrophilic (water soluble) or hydrophobic (lipids soluble) (Maritim et al, 2002). Some of the antioxidant metabolites are described as under:

\section{Uric Acid:-}

Uric acid (UA) is a waste product, formed during breakdown of nucleic acid. It is an oxypurine formed through xanthine by xanthine oxidase. Further oxidation of uric acid to allantoin is catalyzed by the enzyme urate oxidase in about all land animals, but in humans and higher animals, processing of uric acid is not further proceeded due to non-functional uric acid gene. Studies suggest that urate function as an antioxidant by extenuating the oxidative stress originated by high-altitude hypoxia (Strazzullo et al, 2007).

\section{Ascorbic Acid:-}

Ascorbic acid (vitamin C) is an antioxidant compound present in animals and plants species. Human beings can't synthesize ascorbic acid in the body and depend on the dietary source, as the enzyme required to synthesize ascorbic acid has been vanished by means of mutation through the process of human evolution. (Linster et al, 2007).In cells, it is retained in its reduced state by reaction with glutathione. Ascorbic acid is a reducing agent which counteracts the oxidative effect of ROS (Padayatty et al, 2003).

\section{Glutathione:-}

Glutathione (GSH) present in the majority types of aerobic life and cysteine containing tri-peptide. It is not obligatory to take in the diet, since it is produced in the cell by its essential amino acids. It has antioxidant characteristics because in its cysteine moiety the thiol group is a reducing mediator which can be reversibly oxidized or reduced. Glutathione uphold its reduced state in the cell by means of the enzyme glutathione reductase, which in sequence diminish oxidants, metabolites and enzyme systems.

\section{Melatonin:-}

Melatonin is a potent antioxidant which can pass through cell membranes and the blood-brain barrier easily. In contrast with other antioxidants, melatonin doesn't go through redox cycling (the capability of a molecule to go through recurring oxidation and reduction). Melatonin, once oxidized, could not be reduced to its earlier condition since it makes several steady end-products. For that reason, it is termed as a terminal (or suicidal) antioxidant (Tan Dun-Xian et al, 2000).

\section{Tocopherols and Tocotrienols (Vitamin E):-}

Vitamin $\mathrm{E}$ is the combined name for a collection of eight interrelated tocopherols and tocotrienols; these are fatsoluble vitamins containing antioxidant characteristics. Tocotrienols might be crucial in protecting neurons from being damage (Herrera et al, 2001). The probable mechanism of action of antioxidants was first explored when it was recognized that a substance with anti-oxidative action is likely to be the one that itself oxidized voluntarily. Research into how vitamin E help to prevents the process of lipid peroxidation lead to the identification of 
antioxidants as dropping agents that prevent oxidative reactions, oftenly by scavenging reactive oxygen species prior to their damaging effect on the cell (Atkinson et al, 2008).

\section{Antioxidant Enzymes:-}

In hyperglycemic state, oxidative stress stimulates a variety of antioxidant enzyme systems to defend the body tissues from damages. In the body a great number of antioxidant enzymes are found, including:

- Catalase (CAT)

- Superoxide Dismutase (SOD)

- Glutathione (GSH)

- Glutathione Reductase (GR)

- Glutathione Peroxidase (GPX)

- Glutathione-S-transferase (GST)

\section{Catalase (CAT):-}

Catalase was first discovered by Louis Jacques Thenard in 1818. It is an intracellular enzyme formed by four polypeptide chains with four porphyrinheme groups. Catalase role is linked with hydrogen peroxide $\left(\mathrm{H}_{2} \mathrm{O}_{2}\right)$ detoxification in the cells decomposition of hydrogen peroxide $\left(\mathrm{H}_{2} \mathrm{O}_{2}\right)$ to water and oxygen. $\mathrm{H}_{2} \mathrm{O}_{2}$ is a by-product of usual cellular respiration and is also created from superoxide anions by superoxide dismutase action. The enzyme catalyzes the $\mathrm{H}_{2} \mathrm{O}_{2}$ conversion to $\mathrm{H}_{2} \mathrm{O}$ and $\mathrm{O} 2$, using an iron or manganese as a cofactor. In most eukaryotic cells this protein is localized into peroxisomes. Some findings proposed that at low concentrations $\mathrm{H}_{2} \mathrm{O}_{2}$ act as a cellular messenger in insulin signaling, while at high concentrations it is lethal, mainly in pancreatic cells, which are Catalase deprived. Erythrocyte Catalase is the main controller of hydrogen peroxide metabolism (Chelikani et al, 2004).It is also known that in diabetic patients, autoxidation of glucose results in the formation of hydrogen peroxide $\left(\mathrm{H}_{2} \mathrm{O}_{2}\right)$ which also enhances inhibition of antioxidant enzymes (Farah Jabeen et al, 2013).

\section{Superoxide Dismutase (SOD):-}

Superoxide dismutases (SODs) (EC 1.15.1.1) are a class of closely associated enzymes catalyzing the dismutation or breakdown of superoxide anion into oxygen and hydrogen peroxide. It is present in approximately all aerobic cells and in extracellular fluids. It is a fundamental antioxidant enzyme encompasses an antitoxic effect in opposition to superoxide anion. The excess production of SOD may be an adaptive response, resulting in augmented dismutation of superoxide to $\mathrm{H}_{2} \mathrm{O}_{2}$ (Kauret al, 2008). The superoxide radical $\left(\mathrm{O}_{2^{-}}\right)$dismutation into $\mathrm{H}_{2} \mathrm{O}_{2}$ and elemental oxygen (O2) offers a significant defense against the superoxide radical toxicity.

Numerous reports regarding the SOD activity in diabetes mellitus are controversial. Some of the studies give you an idea about an increase in the SOD activity which might be, due to compensatory act of enzyme SOD providing resistance to oxidative stress in diabetics (Farah Jabeen et al, 2012).

SOD exists in three different isoforms (SOD-1, SOD-2 and SOD-3) (Zelkoet al, 2002). There are three families of SOD with respect to the metal cofactor:

$>\mathrm{Cu}-\mathrm{Zn}$ ( binds both copper and zinc)

$>$ Fe or Mn types ( bind either iron or manganese)

$>$ Ni type ( binds nickel)

\section{Copper and Zinc SOD:-}

$\mathrm{Cu}-\mathrm{Zn}$, which is capable to bind with both $\mathrm{Cu}$ and $\mathrm{Zn}$, exists frequently in the cytosol and converts superoxide to hydrogen peroxide. The $\mathrm{Cu}-\mathrm{Zn}$ enzyme is a homodimer (molecular weight of 32,500). The copper and zinc ligands are in histidine side chains (Tainer et al, 1983).

\section{Iron and Manganese SOD:-}

Several prokaryotes posses a type of the enzyme with iron (Fe-SOD), others Mn-SOD and some have both (such as E. coli). Fe-SOD may be found in the plant plastids. Mn-SOD found in human mitochondria. The Mn and Fe SOD active sites have the similar type of amino acid side chains. The manganese ions ligands are an aspartate side chain, 3 histidine side chains with water molecule or OH ligand, based on Mn oxidation state (Borgstahl et al, 1992).

\section{Nickel SOD:-}

Nickel -SOD has a hexameric arrangement make from right-handed 4-helix bundles, each bundle hold N-terminal end chelating a Ni ion. The Ni-hook encloses the motif of His-Cys-X-X-Pro-Cys-Gly-X-Tyr, offering most of the 
contacts significant for metal binding and catalysis and hence Ni-SODs are possibly be utilized for diagnosis (Barondeau et al, 2004).Studies are currently being conducted to realize the potential of SOD as an anti-aging rehabilitation. SOD assists the body to utilize $\mathrm{Zn}, \mathrm{Cu}$, and $\mathrm{Mn}$. Each form of SOD participates a different function in keeping cells healthy (Johnson et al, 2005). Cu-Zn SOD defends the cytoplasm of the cell, and Mn -SOD protects mitochondria of cell from free radical harm. SOD has lots of therapeutic applications (Mates et al, 1999). SOD has been utilized for the management of prostate problems, corneal ulcers, arthritis, burn injuries, inflammatory diseases, and long-term damage by contact to radiation and smoke and to prevent cancer drugs contra-indication (Campana et al, 2004).In its relevant form, it might help to lessen facial wrinkles (Niwa et al 1989), tissue scar, healing burns and wounds, lighten hyper pigmentation, and protect against harmful UV rays (Johnson et al, 2005).

\section{Glutathione System:-}

Glutathione is foremost security system, which avoid oxidation and demolish free radicals. The glutathione system consist of glutathione (GSH), glutathione reductase (GR), glutathione peroxidases (GPX) and glutathione $S$ transferases (GST). This enzyme system is present in microorganisms, plants and animal kingdom whose actions contribute to eliminate superoxide, hydrogen peroxide and hydroxyl radicals (Soto et al, 2003).

\section{Glutathione (GSH):-}

Glutathione is a very important tripeptide antioxidant, having three amino acids specifically, cysteine, glycine and glutamic acid. It is believed as one of the chief non-protein antioxidants in the cell and has been referred to as "master antioxidant." of the body. Glutathione deactivates hydrogen peroxide, lipid peroxides, and peroxynitrite. It also shows powerful anticancer and antiviral properties. It performs an imperative role in the body's immune role via WBCs as well as maintains the integrity of RBCs (Meister et al, 1988).

\section{Glutathione Reductase (GR):-}

This enzyme is a homodimeric flavoprotein, having a central enzymatic role in cellular antioxidant defense system that transforms oxidized glutathione or glutathione disulphide (GSSH) to its reduced sulfhydryl form (GSH). Glutathione found exclusively in its reduced state (GSH). The GR turn activated by means of oxidative stress. Reduced glutathione to oxidized glutathione ratio could be employed to detect the cellular toxicity (Evans et al, 2002).

\section{Glutathione Peroxidase (GPX):-}

Glutathione Peroxidase (GPX) (EC 1.11.1.9) one more substantial antioxidant enzyme found in humans. It is related to a set of antioxidant seleno-enzymes catalyze the reduction of lipid hydroperoxides that defends the cells from damage. GPX levels in the body are in close involvement with glutathione which is the extremely imperative antioxidant found in cells's cytoplasm (Waggiallah et al, 2011).

The fidelity of the cellular and subcellular membranes depends primarily on selenium dependent GPX enzyme. GPX antioxidant effect also defends heart from being damaged by oxidative stress. GPX and GR are found in cytoplasm, mitochondria, and nucleus. GPX metabolizes $\mathrm{H} 2 \mathrm{O} 2$ to water by means of reduced glutathione as hydrogen donor (Maritim et al, 2003).

\section{Glutathione-S-Transferases (GST):-}

Glutathione-S-transferase considered as the set of enzymes catalyzing a range of vital reactions inside the body. It catalyzes the conjugation of GSH via its sulphydryl group to electrophilic centers. The activity of this enzyme is accountable for detoxification of compounds such as lipid peroxides. It has been established that GSTM1, a part of glutathione S-transferase family, participates in detoxification of xenobiotics metabolites involved in cancer. GSTM1 gene deletion consequences in lack of GSTM1 enzyme activity which is related with lung, bladder, prostate, and other tumors. The glutathione-S-transferases demonstrate elevated activity with lipid peroxides. These enzymes are found chiefly in liver and serve its role in detoxification of metabolites (Sharma et al, 2004).

Amplified oxidative stress is extensively accepted contributor in the development and progression of diabetes and its complications. Usually diabetes is accompanied by greater production of reactive oxygen species (free radical) or impaired antioxidant defenses. NADPH dependent reduction of oxidized glutathione is catalyzed by the Glutathione reductase (GR) enzyme that serves to keep up intracellular glutathione supplies and a favorable redox status (Farah Jabeen et al, 2013). 


\section{Lipid Peroxidation AndHyperglycemia:-}

Lipid peroxidation is the oxidative break of lipids. It is the mechanism in which free radical "accept" electrons from lipids of the cell membranes, cause cell damage. This process carries on by a free radical chain reaction. It most oftenly affects polyunsaturated fatty acids (PUFA), as they hold many double bonds in between methylene -CH2groups that grasp especially reactive hydrogens. This radical reaction comprises of three major steps: Initiation, Propagation and Termination (Marnett et al, 1999).

\section{Initiation:-}

Initiation is the initial step in which a fatty acid radical is formed. The most important originators in living cells are ROS such as $\mathrm{OH}$. and $\mathrm{HO} 2$, which act in response of hydrogen atom to form $\mathrm{H}_{2} \mathrm{O}$ and a fatty acid radical (Kanner et al, 1987).

\section{Propagation:-}

The fatty acid radical is an extremely unsteady molecule; consequently it readily reacts with molecular oxygen, generating a peroxyl-fatty acid radical. This is also an unsteady species that reacts with additional free fatty acid. By reacting itself, a fatty acid radical and lipid peroxide or cyclic peroxide is generated. This cycle carry on once, since the newly formed fatty acid radical reacts in the similar mode (Bruce-Keller et al, 1998).

\section{Termination:-}

As a radical reacts with a non-radical, it constantly generates further radicals so that the course is known as "chain reaction system." The radical reaction finishes, once the two radicals react and make a non-radical species. This occurs only when the level of radical species is elevated sufficient to have a greater chance of collision of two radicals (Bruce-Keller et al, 1998).Living organisms have developed diverse molecules that accelerate termination and hence defending the cell membrane. There are many factors contributing in lipid peroxidation (Imlay et al, 2003).

Free radicals are extremely reactive chemical group that bring oxidative injury to the living beings by attacking the macromolecules including carbohydrates, proteins, lipids and nucleic acids. Under normal physiological state, there is a critical balance in the generation of oxygen free radicals and antioxidant defense systems used by organisms to deactivate and protect themselves against free radical toxicity (Etsuo et al, 2005).

Oxidant/antioxidant equilibrium disturbance creates a state known as oxidative stress. The incidence of free radical provoke lipid peroxidation brings significant alteration in the cell membrane (Agarwal et al, 1985). Membrane lipid peroxidation has been linked to the pathogenesity of various degenerative diseases, such as atherosclerosis, carcinogenesis, sickle cell anemia, aging and Diabetes Mellitus (Chattergee et al, 1988). For that reason, the lipid peroxide in the blood offers valuable information for the prediction of diabetes where secondary disorders are often lethal (Tappel et al, 1973). Free radicals possibly play a key role in the cause and impediment of diabetes mellitus. In DM, endogenous free radical scavenge protection mechanisms amendment may direct the unsuccessful scavenging of ROS, inevitably cause oxidative damage and tissue injury (Suckling et al, 1993). If this process is not finished quickly enough, there will be harm to the cell membrane, which primarily comprises of lipids. Additionally, lipid peroxidation end-products might be mutagenic and carcinogenic (Laura et al, 2003). Certain diagnostic tests are accessible for the quantification of the lipid peroxidation end-products, particularly malondialdehyde (MDA) (Janero et al, 1990). The most frequently exploited test is called TBARS Assay (thiobarbituric acid reactive substances assay).

The redox imbalance causes the activation of stress-sensitive intracellular signaling pathways that play a key role in the development of late complications of diabetes mellitus, along with mediation in insulin resistance and impaired insulin secretion. Insulin affects many sites of mammalian lipid metabolism. The incidence of free radical induced lipid peroxidation causes considerable change in the cell membrane (Agarwal et al, 1985). Hyperglycemia induces the production of ROS causing oxidative stress as well as lipid peroxidation which may accelerate vascular complication in diabetics (Farah Jabeen et al, 2014). From this point of view the assessment of various lipid fractions and lipid peroxides in cases of Diabetes Mellitus may be of some help in the prognosis of patients and in preventing the possibilities of complications or secondary disorders (Suryawanshi et al, 2006). By measuring the MDA level and by supplementing the antioxidants accordingly the physician could manage the severity of micro vascular complications of diabetic subjects (Shih et al, 1997). 


\section{Antioxidant Therapy in Hyperglycemia:-}

During the past decade, a number of clinical trials of antioxidants were performed on diabetic patients with a view to compete the oxidative stress and to determine their possible use as therapeutic agents. Vitamin $\mathrm{E}$ and vitamin $\mathrm{C}$ were mostly studied, but the results were not supportive in all patients and often more than one treatment was needed to achieve a desired results. Type 2 diabetic patients responded well to vitamin E. The use of choosy antioxidants beside with indispensable trace elements and minerals might be effective in reducing deleterious effects of oxidative stress in diabetic patients (Maritim et al, 2002) .Further studies are required in this direction.

\section{Conclusion:-}

Enhanced production of free radicals, reactive metabolites and oxidative stress is central event to the development and progression of diseases. These metabolites are continuously controlled by endogenous antioxidant enzyme systems, which may be enzymatic or non-enzymatic or transition metals. Thus, the balance is created between prooxidants and antioxidants. Furthermore increase in the levels of oxygen and nitrogen free radicals (ROS/RNS) has been linked with lipid peroxidation, non-enzymatic glycation of proteins and oxidation of glucose that causes considerable change in the cell membrane which contributes toward diabetes mellitus and its related micro and macro vascular complications. Most of the studies have shown relationship between oxidative stress and diabetes along with their complications related to heart, liver kidney and eye. The impairment of antioxidant status, either by exogenous or endogenous sources, may disturb the cellular redox balance and the pathological conditions would be the main characteristics and forms oxidative stress in cells or tissues.It has been found that persistent hyperglycemia produces havoc in the whole body system by altering various metabolic processes leading to abnormal lipid profile, glycation of proteins and lipoproteins, per oxidation of membrane lipids, activation of oxidative stress by disturbing oxidant/antioxidant balance, inhibition of antioxidant enzymes and structural and functional abnormalities in small and large blood vessel leading to vascular damage. Antioxidants have shown to be potential in the treatment of both type 1 and type 2 diabetes. Therefore, it seems reasonable that antioxidants can play an important role in the improvement of diabetes. The use of selective antioxidants beside with essential trace elements and minerals might be effective in reducing deleterious effects of oxidative stress in diabetic patients.It is very important to understand that increased antioxidants could disrupt the normal biological oxidant processes, and consequently terminate the reactive metabolites to perform their normal biological roles, which may trigger the precancerous substances. Further studies are required in this direction.

\section{References:-}

1. Agarwal S, Banerjee S, Chatterjee SN.(1985): Effects of oxygen on ferrous sulphate induced lipid peroxidation in liposomal membrane. Indian J Biochem Biophys; 22(6): 331-334.

2. Atkinson; Epand, RF; Epand, RM .(2008):Tocopherols and tocotrienols in membranes: A critical review. Free radical Biol Med.; 44 (5): 739-764.

3. Barondeau DP, Kassmann CJ, Bruns CK, Tainer JA, Getzoff ED.( 2004): Nickel superoxide dismutase structure and mechanism. Biochemistry.; 43(25): 8038-8047.

4. Baynes JW, Thorpe SR.(1999): Role of oxidative stress in diabetic complications: A new perspective on an old paradigm. Diabetes.; 48: 1-9.

5. Baynes JW.(1991): Role of oxidative stress in development of complications in diabetes. Diabetes; 40: 405-412.

6. Bierhaus A, Hofmann MA, Ziegler R., Nawroth PP.(1998): AGEs and their interaction with AGE- receptors in vascular disease and diabetes mellitus. I. The AGE concept. Cardiovasc. Res.; 37: 586-600.

7. Borgstahl GE, Parge HE, Hickey MJ, Beyer WF Jr, Hallewell RA, Tainer JA.(1992): The structure of human mitochondrial manganese superoxide dismutase reveals a novel tetrameric interface of two 4-helix bundles. Cell.; 71: 107-118.

8. Boullier A, Bird DA, Chang MK, Dennis EA, Friedman P, Gillotre-Taylor K, (2001) :Scavenger receptors, oxidized LDL and atherosclerosis. Ann. N.Y. Acad. Sci.; 947:214-222.

9. Brownlee M, Cerami A, Vlassara H.( 1998): Advanced glycosylation end product in tissue and the biochemical basis of diabetic complications. N Engl J Med.; 318: 1315-1321.

10. Bruce-Keller AJ, Li YJ, Lovell MA, Kraemer PJ, Gary DS, Brown RR, Markesbery WR, Mattson MP.( 1998) 1998: 4-Hydroxynonenal, a product of lipid peroxidation, damages cholinergic neurons and impairs visiospatial memory in rats. J NeuropatholExp Neurol.; 57: $257-267$.

11. Campana F, Zervoudis S, Perdereau B, Gez E, Fourquet A, Badiu C, Tsakiris G, Koulaloglou S. (2004): Topical superoxide dismutase reduces post-irradiation breast cancer fibrosis. J Cell Mol Med.; 8 (1): 109-116. 
12. Ceriello A. (2000): Oxidative stress and glycemic regulation, Metabolism.; 49(2): 27-29.

13. Chattergee SN, Agrawal S, Amit K. (1988): Membrane lipid peroxidation and its pathological consequence. Ind. J. of Biochem and Biophys.; 25: 31.

14. Chelikani P, Fita I and Loewen PC. (2004): Diversity of structures and properties among catalases. CMLS, Cell. Mol. Life Sci.; 61: 192 - 208.

15. Davi G, Falco A, Patrono C. (2005): Lipid peroxidation in diabetes mellitus. Antioxid. Redox Signal.; 7: 256268.

16. Dawson J and Walters M. (2006): Uric acid and xanthine oxidase: Future therapeutic targets in the prevention of cardiovascular disease? Br J Clin Pharmacol.; 62(6): 633-644.

17. DCCT Research Group. (1993): The effect of intensive treatment of diabetes on the development and progression of long-term complications in Insulin-Dependent Diabetes Mellitus. N EnglJ Med.; 329: $977-986$.

18. Etsuo N, Yasukazu Y, Saito Y, Noguchi N. (2005): Lipid peroxidation: Mechanisms, inhibition, and biological effects. Biochem Biophys Res Commun.; 338: 668-676.

19. Evans JL. Goldfine ID. Maddux BA. Grodsky GM. (2002): Oxidative stress and stress-activated signaling pathways: A unifying hypothesis of type 2 diabetes. Endocr Rev.; 23:599-622.

20. Farah Jabeen, Asher Fawwad, Husan Afroz Rizvi, Faraz Alvi, Farha Aziz. (2014) :Evaluation of Lipid peroxidation Marker (MDA), C - reactive protein, and leucocytes indices as Inflammatory Markers in Patients with Type 2 Diabetes Mellitus. International Journal of Advanced Research. 2 (1): 303-309.

21. Farah Jabeen, Husan Afroz Rizvi and Anum Subhan.(2012): Effect of Hyperglycemia o Superoxide Dismutase Defense System and Erythrocyte Indices in Diabetic Patients. Pakistan journal of Biochemistry and Molecular biology.;45: (2).

22. Farah Jabeen, Husan Afroz Rizvi, Farha Aziz, Yasmin Anjum (2013):Effect of Glycemic Control on Lipid Profile, Platelet Indices and Antioxidant Enzymes (Catalase and Superoxide dismutase Activities in Type 2 Diabetics. International Journal of Advanced Research. 1(7), 207-215.

23. Farah Jabeen, Husan Afroz Rizvi, Rafia Azmat, Farha Aziz. (2013): Impairment of Glutathione metabolism and its impact on other Biochemical constituents in Patients of Diabetes Mellitus" Pak. J. Biochem. Mol. Biol.,; 46(3): 92-96.

24. Fitzpatrick FA. (2004): Cyclooxygenase enzymes: Regulation and function. Curr Pharm Des.; 10(6): $577-588$.

25. Gillery P. (2001): Advanced glycation end products (AGES), free radicals and diabetes. J SocBiol; 195(4): 387390.

26. Grobbee DE.(2003) How to advance prevention of cardiovascular complications in type 2 diabetes. Metabolism.; 52(1):24-28.

27. Guzik TJ, Mussa S, Gastaldi D, Sadowski J, Ratnatunga C, Pillai R, Channon KM. (2002): Mechanisms of increased vascular superoxide production in human diabetes mellitus: Role of NAD(P)H oxidase and endothelial nitric oxide synthase. Circulation. 105(14): 1656-1662.

28. Haffner SM.(1998) Management of dyslipidemia in adult with diabetes. Diabetes Care.; 21:1600-1678.

29. Harris MI,( 1995): National Diabetes Data Group. National Institute of Health, Diabetes Digestive and Kidney Diseases. Diabetes in America, 2nd Ed., NIH (Publication No. 95-1468).:293-338.

30. Harrison R.(2002): Structure and function of xanthine oxidoreductase: where are we now? Free Radic. Biol. Med.; 33 (6):774-797.

31. Herrera E, Barbas C.(2001): Vitamin E: Action, metabolism and perspectives. J PhysiolBiochem.; 57(2): 43-56.

32. Hille R.(2005): Molybdenum-containing hydroxylases. Arch. Biochem. Biophys.;433(1): $107-116$.

33. Ido Y, Kilo C, Williamson JR.(1997): Cytosolic NADH/NAD+, free radicals, and vascular dysfunction in early diabetes mellitus. Diabetologia.; 40 (2): S115-S117.

34. Imlay JA. (2003): Pathways of oxidative damage. Annual Review of Microbiology. 57: 395-418.

35. Inoguchi T, Sonta T, Tsubouchi H, Etoh T, Kakimoto M, Sonoda N, et al. (2003): Protein Kinase C-dependent increase in Reactive Oxygen Species (ROS) production in vascular tissues of diabetes: Role of vascular NAD(P)H oxidase. J Am SocNephrol.; 14: 227-232.

36. Jacob RA.(1995): The Integrated Antioxidant System. Nutr Res.; 15(5): 755-766.

37. Janero DR. (1990): Malondialdehyde and thiobarbituric acid-reactivity as diagnostic indices of lipid peroxidation and peroxidative tissue injury. Free Radic. Biol. Med.; 9: 515-540.

38. Jiang F, Zhang Y and Dusting GJ. (2011): NADPH Oxidase-mediated redox signaling: Roles in Cellular stress response, stress tolerance, and tissue repair. Pharmacological Reviews.; 63 (1): 218-242.

39. Johansen S J, Alex HK, Rychly DJ and Ergul A. (2005): Oxidative stress and the use of antioxidants in diabetes: Linking basic science to clinical practice. Cardiovascular Diabetology. 4: 5-12. 
40. Johnson F, Giulivi C.(2005): Superoxide dismutases and their impact upon human health. Mol Aspects Med.; 26 (4-5):340-352.

41. Kanner J, German JB, Kinsella JE. (1987): Initiation of lipid peroxidation in biological systems. Crit Rev Food SciNutr.; 25(4): 317-364.

42. Kaur K., Bedi G, Kaur M, Anil Vij, Kaur I. (2008) Lipid peroxidation and the levels of antioxidant enzymes in coronary artery disease. Indian J Clin Biochem.; 23(1): 33-37.

43. Klein R.(1995): Hyperglycemia and microvascular and macrovascular disease in diabetes. Diabetes Care.; 18(2):258-268.

44. Laura J, Niedernhofer J, Daniels S, Rouzer CA, Greene RE, Marnett L J. (2003): Malondialdehyde, a Product of lipid peroxidation, is mutagenic in human cells. J Biol Chem.; 278: 31426- 31433.

45. Lenaz G. (2001): The mitochondrial production of reactive oxygen species: Mechanisms and implications in human pathology. IUBMB Life,; 52(35): 159-164.

46. Linster, CL, Van Schaftingen, E. (2007): Vitamin C. FEBS J.; 274 (1): 1-22.

47. Maritim AC, Sanders RA, Watkings JB. (2002): Diabetes, Oxidative Stress and Antioxidants. J BiochemMolToxicol.; 17(1): 2-4.

48. Maritim AC, Sanders RA, Watkins JB. (2003): Effects of alpha-lipoic acid on biomarkers of oxidative stress in streptozotocin-induced diabetic rats. J Nutr Biochem.; 14: 288-294.

49. Marnett LJ. (1999): Lipid peroxidation-DNA damage by malondialdehyde. Mutation research. 424(1-2): 83-95.

50. Mates JM, Perez-Gomez C and Nunez de Castro I. (1999): Antioxidant enzymes and human diseases. Clin. Biochem.: 32, 595-603.

51. Meister A. (1988): Glutathione metabolism and its selective modification. J Biol Chem.; 263(17): 205-208.

52. Meyer C, Dostou JM, Welle SL, Gerich JE. (2002): Role of human liver, kidney, and skeletal muscle in postprandial glucose homeostasis. Am J PhysiolEndocrinolMetab.; 282: 419-427.

53. Mironova MA, Klein RL, Virella GT, Lopes-Virella MF. (2000): Modified LDL antibodies, LDL- containing immune complexes, and susceptibility of LDL to in vitro oxidation in patients with type 2 diabetes. Diabetes. 49: 1033-1041.

54. Mullarkey CJ, Edelstein D, Brownlee M. (1990): Free radical generation by early glycation products: A mechanism for accelerated atherogenesis in diabetes. BiochemBiophys Res Commun.; 173(3): 932-939.

55. Niedowicz DM, Daleke DL. (2005): The role of oxidative stress in diabetic complications. Cell Biochem Biophys.; 43(2): 289-330.

56. Nishikawa T, Edelstein D, Du XL, Yamagishi S, Matsumura T, Kaneda Y, et al. (2000): Normalizing mitochondrial superoxide production blocks three pathways of hyperglycaemic damage. Nature. 404:787-790.

57. Niwa Y. (1989): Lipid peroxides and superoxide dismutase (SOD) induction in skin inflammatory diseases and treatment with SOD preparations. Dermatologica.; 179 (1):101-106.

58. Padayatty SJ, Katz A, Wang Y, Eck P, Kwon O, Lee JH, et al. (2003): Vitamin C as an antioxidant: Evaluation of its role in disease prevention. J Am CollNutr.; 22: 18-35.

59. Patel A, MacMahon S, Chalmers J, Neal B, Billot L, etal. (2008): Intensive blood glucose control andvascular outcomes in patients with type 2 diabetes. N Engl J Med.; 358(24): 2560-2572.

60. Pazdro RR and Burgess JR. (2010): The role of vitamin E and oxidative stress in diabetes complications. Mech Ageing Dev.; 131(4): 276-286.

61. Rains JL, Jain SK.(2011): Oxidative stress, insulin signaling, and diabetes. Free RadicBiol Med.; 50(5): 567 575.

62. Rains JL, Jain SK. (2011): Oxidative stress, insulin signaling, and diabetes. Free RadicBiol Med.; 50(5): 567575.

63. Rhee SG. (2006): Cell Signaling: $\mathrm{H}_{2} \mathrm{O}_{2}$, a necessary evil for cell signaling. Science. 312 (5782): 1882-1883.

64. Sakurai T, Tsuchiya S. (1988): Superoxide production from non-enzymatically glycated protein. FEBS Lett .; 236: 406-410.

65. Setter SM, Campbell RK, Cahoon CJ. (2003): Biochemical pathways for microvascular complications of diabetes mellitus. Ann Pharmacother.; 37(12):1858-1866.

66. Sharma R, Yang Y, Sharma A, Awasthi S, Awasthi Y. (2004): Antioxidant role of glutathione S transferases: Protection against oxidant toxicity and regulation of stress-mediated apoptosis. Antioxid Redox Signal.; 6 (2): 289-300.

67. Shih KC, Kwak CF, Hwa CM. (1997): Acipimox attenuates hyper triglyceredemia in Dislipidemic Non-insulin dependent diabetes mellites patients without perturbation of insulin sensitivity and glycemic control. Diabetic. Res. clin. Pract.; 36(2): 113-119.

68. Sies H. (1997): Oxidative stress: oxidants and antioxidants. Exp Physiol.; 82(2):291-295. 
69. Sies H. (1993): Strategies of antioxidant defense. Eur J Biochem.; 215:213-219.

70. Soto C, Recoba R, Barron H, Alvarez C, Favari L. (2003): Silymarin increases antioxidant enzymes in alloxaninduced diabetes in rat pancreas. Comp Biochem Physiol.;136:205-212.

71. Strazzullo P, Puig J. (2007): Uric acid and oxidative stress: Relative impact on cardiovascular risk. Nutr Metab Cardiovas Diseases.; 17 (6): 409-414.

72. Suckling KE, Brian J. (1993): Animal models of human lipid metabolism. Prog. Lipid. Res.; 32:124.

73. Suryawanshi NP, Bhutey AK, Nagdeote AN, Jadhav AA, Manoorkar GS. (2006): Study of lipid peroxide and lipid profile in diabetes mellitus. Indian J ClinBiochem.; 21(1): 126-130.

74. Suzuki D, Miyata T, Saotome N, Horie K, Inagi R, Yasuda Y, et al. (1999): Immuno-histochemical evidence for an increased oxidative stress and carbonyl modification of proteins in diabetic glomerular lesions. J Am SocNephrol.; 10(4): 822-832.

75. Tainer JA. (1983): Structure and mechanism of copper, zinc superoxide dismutase. Nature.; (5940): 284-287.

76. Tan Dun-Xian, Manchester, Lucien C, Reiter, Russel J, Qi, Wen-Bo, Karbownik, Malgorzata, Calvo, Juan R. (2000): Significance of melatonin in antioxidative defense system: Reactions and products. Neurosignals; 9 (34): $137-159$.

77. Tappel AL, (1973): Lipid peroxidation damage to cell components. ClinPathol. Fed Proce.;32(8): 1870-1874.

78. Thompson CS. (2008): Animal models of diabetes mellitus: Relevance to vascular complications. Curr Pharm Des.; 14: 309-324.

79. Van Dam PS. (2002): Oxidative stress and diabetic neuropathy: Pathophysiological mechanisms and treatment perspectives. Diabetes Metab Res Rev.; 18: 176-184.

80. Vincent AM, Russell JW, Low P, Feldman EL. (2004): Oxidative stress in the pathogenesis of diabetic neuropathy. Endocr Rev.; 25: 612-628.

81. Waggiallah H, Alzohairy M. (2011): The effect of oxidative stress on human red cells glutathione peroxidase, glutathione reductase level and prevalence of anemia among diabetics. North Am J Med Sci.; 3(7): 344-347.

82. Wolff SP, Jiang ZY, Shunt JV. (1991): Protein glycation and oxidative stress in diabetic mellitus and aging. Free Rad. Biol. Med.; 10: 339-352.

83. Xia P, Inoguchi T, Kern TS, Engerman RL, Oates PJ, King GL. (1994): Characterization of the mechanism for the chronic activation of diacylglycerol-protein kinase $\mathrm{C}$ pathway in diabetes and hypergalactosemia. Diabetes.; 43: $1122-1129$.

84. Yamagishi S, Imaizumi T. (2005): Diabetic vascular complications: Pathophysiology, biochemical basis and potential therapeutic strategy. Curr Pharm Des.; 11: 2279-2299.

85. Zelko IN, Mariani TJ, Folz RJ. (2002): Superoxide dismutase multigene family: A comparison of theCuZnSOD (SOD1), Mn-SOD (SOD2), and EC-SOD (SOD3) gene structures, evolution, and expression. Free Rad Biol Med.; 33: 337-349. 